\title{
Efficacy and safety of ustekinumab in Japanese patients with moderately to severely active Crohn's disease: a subpopulation analysis of phase 3 induction and maintenance studies
}

\author{
Toshifumi Hibi ${ }^{1}$, Yuya Imai ${ }^{2}$, Yoko Murata ${ }^{2}$, Nobuko Matsushima ${ }^{2}$, Richuan Zheng ${ }^{2}$, Christopher Gasink ${ }^{3}$ \\ ${ }^{I}$ Center for Advanced IBD Research and Treatment, Kitasato Institute Hospital, Kitasato University, Tokyo, ${ }^{2} J a n s s e n$ Pharmaceutical K.K., Tokyo, \\ Japan, ${ }^{3}$ Janssen Research \& Development, LLC, Spring House, PA, USA
}

Background/Aims: Efficacy and safety of ustekinumab were evaluated in a Japanese subpopulation with moderately to severely active Crohn's disease (CD) in UNITI-1, UNITI-2 and IM-UNITI studies and results were compared with the overall population. Methods: Overall, patients in UNITI-1 (Japan, $\mathrm{n}=56$; failed response to tumor necrosis factor antagonist) and UNITI-2 (Japan, $\mathrm{n}=26$; failed response to prior conventional therapy) were randomized to placebo or ustekinumab intravenous induction ( $130 \mathrm{mg}$ or $\sim 6 \mathrm{mg} / \mathrm{kg}$ ) at week 0 . Responders to ustekinumab induction therapy (Japan, $\mathrm{n}=21$ ) were randomized to placebo or ustekinumab (90 mg, subcutaneous) maintenance (every 12 weeks [q12w] or 8 weeks [q8w]) in IM-UNITI. The primary endpoint was clinical response at week 6 for induction studies and clinical remission at week 44 for maintenance study. Results: Percentage of patients achieving clinical response at week 6 was greater in ustekinumab $130 \mathrm{mg}$ and $\sim 6 \mathrm{mg} / \mathrm{kg}$ groups than in the placebo group (UNITI-1: $36.8 \%$ and $31.6 \%$ vs. $27.8 \%$, respectively, for Japanese; $34.3 \%$ and $33.7 \%$ vs. $21.5 \%$, respectively, for overall; UNITI-2: $37.5 \%$ and $55.6 \%$ vs. $11.1 \%$, respectively, for Japanese; $51.7 \%$ and $55.5 \%$ vs. $28.7 \%$, respectively, for overall). Clin-

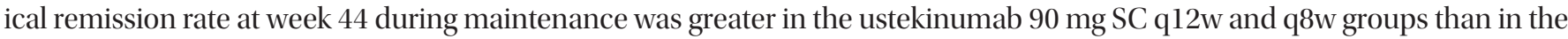
placebo group (50.0\% and 55.6\% vs. $25.0 \%$, respectively, for Japanese; $48.8 \%$ and $53.1 \%$ vs. $35.9 \%$, respectively, for overall). Efficacy and safety results observed in the Japanese subpopulation were generally consistent with those in the overall population. Conclusions: Ustekinumab could be considered as a new therapeutic option for moderately to severely active CD in Japanese patients. Both ustekinumab induction and maintenance treatments were generally well tolerated (Clinical Trial Registration: NCT01369329, NCT01369342, NCT01369355). (Intest Res 2017;15:475-486)

Key Words: Crohn disease; Interleukin-12/23; Japan; Ustekinumab

Received December 31, 2016. Revised March 10, 2017.

Accepted March 10, 2017. Published online June 19, 2017

Correspondence to Yuya Imai, Janssen Pharmaceutical K.K., 5-2 Nishikanda 3-chome, Chiyoda-ku, Tokyo 101-0065, Japan. Tel: +81-80-12566377, Fax: +81-3-4411-5086, E-mail: yimai@its.jnj.com

${ }^{*}$ This paper was presented at 7th Annual Meeting of Japanese Society of Inflammatory Bowel Disease (JBSID) 2016, July 9-10, Kyoto, Japan.

Financial support: This study was funded by Janssen Research \& Development, LLC and Janssen Pharmaceutical K.K., Japan. Conflict of interest: Yuya Imai, Yoko Murata, Nobuko Matsushima, and Richuan Zheng are employees of Janssen Pharmaceutical K.K., Japan. Dr. Christopher Gasink is an employee of Janssen Research \& Development, LLC, United States. Dr. Toshifumi Hibi is a consultant for Janssen Pharmaceutical K.K., Japan.

\section{INTRODUCTION}

Crohn's disease (CD) is a chronic, immune-mediated IBD that is often characterized by remission and recurrent relapses. ${ }^{1,2}$ The incidence of CD has increased over the past 5 decades in developed countries, and it varies from 0 to 20.1 cases per 100,000 persons in North America and from 0.3 to 12.7 cases per 100,000 persons in European countries. ${ }^{3}$ The incidence of $\mathrm{CD}$ in Japan has increased from 0.60 to 1.20 between 1986 and 1998, and although it is much lower than in

\footnotetext{
๑ Copyright 2017. Korean Association for the Study of Intestinal Diseases. All rights reserved.

This is an Open Access article distributed under the terms of the Creative Commons Attribution Non-Commercial License (http://creativecommons.org/licenses/by-nc/4.0)

which permits unrestricted non-commercial use, distribution, and reproduction in any medium, provided the original work is properly cited.
} 
the Western countries, it has rapidly increased in the past 20 years. ${ }^{4,5}$ The changing dietary habits due to urbanization and other environmental risk factors along with the changes in gastrointestinal microbiota may be responsible for the rising incidence of CD in Japan. ${ }^{6-8}$

The current standard of care for CD in Japan consists of corticosteroids for induction and 5-aminosalicylic acid, azathioprine, 6-mercaptopurine, and tumor necrosis factor (TNF) antagonists (infliximab and adalimumab) for induction and maintenance. ${ }^{9,10}$ TNF-antagonists are the only biologic therapies available for patients with moderately to severely active CD in Japan. Although TNF antagonists have advanced the management of $\mathrm{CD}$, a considerable number of patients show primary nonresponse, loss of response, or intolerance to the treatment. ${ }^{11-14}$ Therefore, there is currently an unmet need for additional treatment options with new mechanisms of action for moderately to severely active CD, particularly in Japan.

Ustekinumab is a fully human monoclonal IgG1 antibody that targets the biological activity of interleukin (IL)-12/23 through their shared subunit p40 and inhibits signaling through these 2 cytokine receptors. ${ }^{15}$ Ustekinumab is currently approved for the treatment of psoriasis and psoriatic arthritis in various countries, including Japan, ${ }^{16,17}$ and it is currently approved for the treatment of moderately to severely active CD in the United States and Europe. In a previous phase $2 \mathrm{~b}$ global study conducted in moderately to severely active CD patients who were refractory to TNF antagonists, intravenous (IV) ustekinumab induction therapy showed increased rates of clinical responses at week 6 (36.6\% for $1 \mathrm{mg} / \mathrm{kg}$, 34.1\% for $3 \mathrm{mg} / \mathrm{kg}$, and $39.7 \%$ for $6 \mathrm{mg} / \mathrm{kg}$ vs. $23.5 \%$ for placebo, $P=0.005$ ), and SC ustekinumab maintenance therapy showed increased rates of response $(69.4 \%$ vs. $42.5 \%, P<0.001)$ and remission $(41.7 \%$ vs. $27.4 \%, P=0.03)$ at week $22{ }^{18}$ A phase 3 global clinical study program, comprising of two 8-week induction studies (UNITI-1 and UNITI-2) and a 44-week maintenance study (IM-UNITI), was designed to evaluate efficacy and safety of ustekinumab over a period of 52 weeks in patients with moderately to severely active CD who were refractory to TNF antagonists or conventional therapy. ${ }^{19}$ Here, we report the efficacy and safety of ustekinumab from the UNITI-1, UNITI-2, and IM-UNITI global studies in a Japanese subpopulation.

\section{METHODS}

\section{Study Overview}

The phase 3 ustekinumab development program for CD comprised of two 8-week induction studies (UNITI-1 [NCT01369329] and UNITI-2 [NCT01369342]) and one 44week randomized withdrawal (of ustekinumab-induction responders) maintenance study (IM-UNITI [NCT01369355]) (Fig. 1). The details on the UNITI-1, UNITI-2, and IM-UNITI study designs have been described elsewhere. ${ }^{19}$ All the 3 studies were multicenter, randomized, double-blind, placebo-controlled, and were conducted between July 2011 and June 2015 in various countries, including Japan (13 sites for UNITI- 1 and 16 sites for UNITI-2, with subjects from 20 sites continuing to participate in IM-UNITI).

Patients who were $\geq 18$ years of age with $\geq 3$ months of CD with a CDAI score of 220 to 450 were eligible for induction studies. In UNITI-1 study, patients were required to have had primary or secondary nonresponse or intolerance to $\geq 1$ TNF antagonists. In UNITI-2, patients were required to have failed conventional therapy, and they could have undergone TNF antagonist(s) therapy but should not have demonstrated a primary or secondary nonresponse or intolerance. Treatment with immunosuppressants, mesalamine, antibiotics, and/or oral corticosteroids (prednisone $\leq 40 \mathrm{mg}$ /day or budesonide $\leq 9 \mathrm{mg} /$ day) at stable doses was permitted Patients previously treated with IL-12 or IL-23-antagonists were excluded. Patients with Crohn's complications anticipated to require surgical treatment or that could impede the assessment of treatment response using CDAI, active tuberculosis, and other infections or history of cancer were also excluded. Eligible patients in both induction studies were randomized to receive a single IV dose of placebo or ustekinumab (130 $\mathrm{mg}$ dose) or a weight-based dose of ustekinumab approximating $6 \mathrm{mg} / \mathrm{kg}$ (260 $\mathrm{mg}$ [weight $\leq 55$ $\mathrm{kg}$ ], $390 \mathrm{mg}$ [weight $>55 \mathrm{~kg}$ and $\leq 85 \mathrm{~kg}$ ], or $520 \mathrm{mg}$ [weight $>85 \mathrm{~kg}]$ ) at baseline (week 0).

Patients who responded to ustekinumab IV at week 8 in either of the induction studies were eligible to enter the 44-week maintenance study (IM-UNITI) as the primary (randomized) population. These patients underwent randomized withdrawal, receiving SC administration of either placebo or 1 of 2 ustekinumab maintenance regimens (ustekinumab $90 \mathrm{mg}$ every 12 weeks [q12w] or ustekinumab 90 mg every 8 weeks $[\mathrm{q} 8 \mathrm{w}])$. Patients who did not show a clinical response to ustekinumab induction and all placebotreated patients were eligible to enter IM-UNITI but were not 


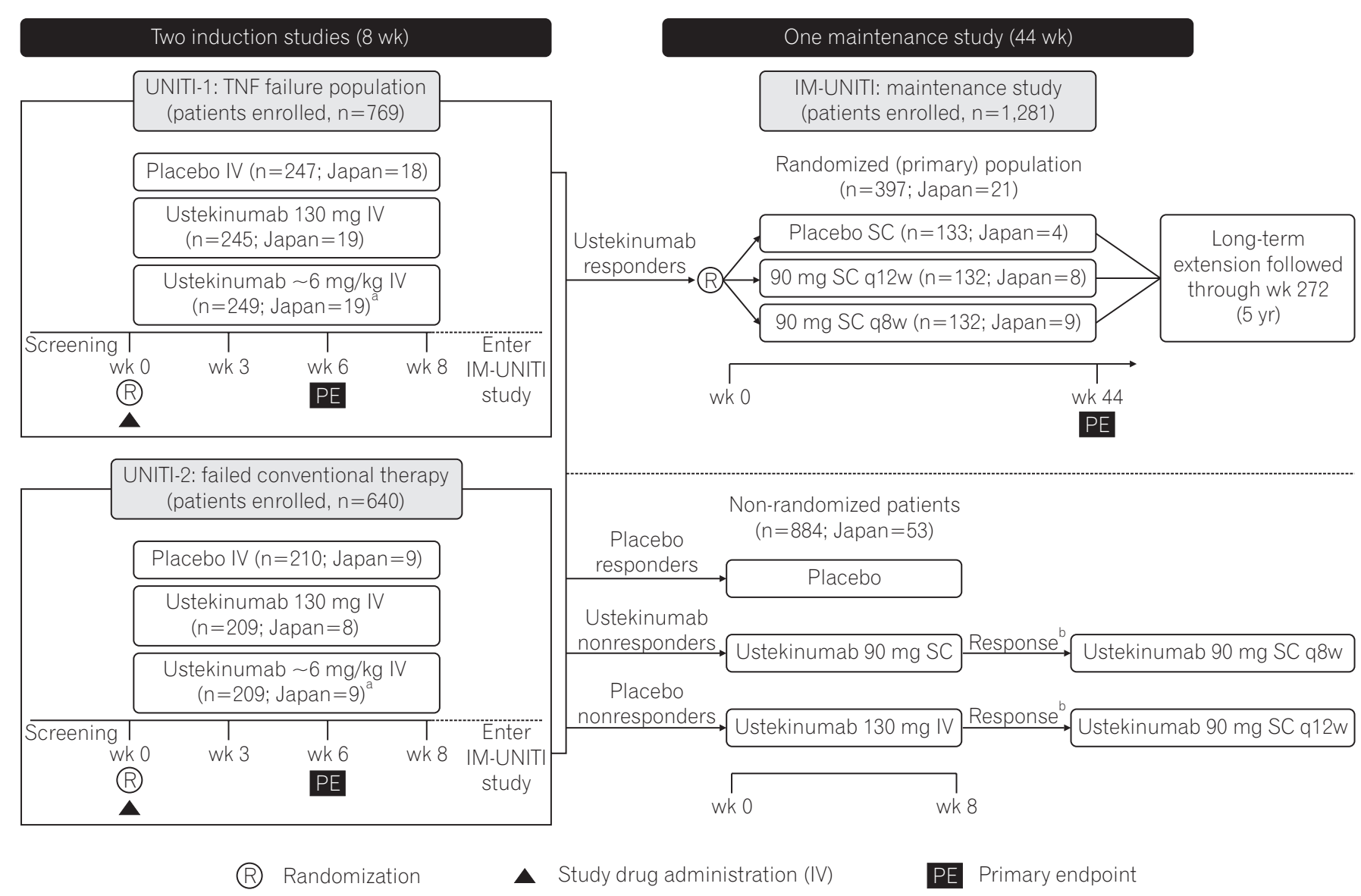

Fig. 1. Study design of phase 3 CD program for ustekinumab. Patients randomized to placebo and patients who were nonresponders to ustekinumab were eligible for nonrandomized maintenance dose after completion of induction study. ${ }^{a}$ Weight range based ustekinumab doses approximating at 6 $\mathrm{mg} / \mathrm{kg}_{;}{ }^{b}$ If there was no clinical response achieved at week 8, the study treatment was discontinued. TNF, tumor necrosis factor; IV, intravenous; q8W, every 8 weeks; q12w, every 12 weeks. Adapted from Feagan BG, et al. N Engl J Med 2016;375:1946-1960. ${ }^{19}$

included in the primary population (Fig. 1).

In all 3 studies, randomization was performed centrally using permuted blocks. Stratification variables in both induction studies included study region, CDAI score ( $\leq 300$ or $>300$ ), and presence/absence of initial response to TNF-antagonist therapy (only in UNITI-1). In IM-UNITI, stratification variables included ustekinumab induction dose received and remission status at maintenance baseline (week 0 ).

The independent ethics committee or institutional review board at each study site approved study protocols. All 3 studies were conducted in accordance with the Declaration of Helsinki, Good Clinical Practices, and applicable regulatory requirements. Written informed consent was obtained from all patients prior to enrollment.

\section{Study Assessments}

The CDAI scores and CRP concentrations were evaluated at weeks $0,3,6$, and 8 in the induction studies and every 4 weeks in the maintenance study. Fecal calprotectin and lactoferrin concentrations were evaluated at weeks 0 and 6 during induction and at weeks 8,24 , and 44 during maintenance. Safety assessments were performed throughout the study, which included monitoring of treatment-emergent adverse events (TEAEs) and vital signs and clinical laboratory evaluations.

Additionally, serum ustekinumab concentrations were measured using a validated electrochemiluminescent immunoassay method at weeks $0,3,6$, and 8 during induction and every 4 weeks during maintenance. Antibodies against ustekinumab were evaluated using drug-tolerant electrochemiluminescent assay at weeks 0 and 6 during induction and at weeks 12, 24, 36, and 44 during maintenance. 


\section{Study Endpoints}

\section{1) Induction Studies}

The primary endpoint was clinical response (defined as a reduction from baseline in CDAI score $\geq 100$ ) at week 6 . Patients with a baseline CDAI score between 220 and 248 were considered to show a clinical response if a CDAI score of $<150$ was attained. The major secondary endpoints were clinical remission at week 8 (defined as a CDAI score of $<150$ ), clinical response at week 8 , and 70-point response (defined as a reduction from baseline in the CDAI score of $\geq 70)$ at weeks 3 and 6 .

\section{2) Maintenance Study}

The primary endpoint was clinical remission at week 44 (defined as a CDAI score of $<150$ ). The major secondary endpoints were clinical response at week 44, clinical remission at week 44 among patients in clinical remission to ustekinumab at week 0 , corticosteroid-free remission at week 44 , and clinical remission at week 44 in the subset of patients who were refractory or intolerant to TNF antagonist therapy (i.e., patients from UNITI-1 induction study).

Other endpoints included change from baseline in CRP, fecal calprotectin, and lactoferrin concentrations during induction and maintenance.

\section{Statistical Analyses}

Statistical analyses for the overall study population have been described elsewhere. ${ }^{19}$ In both induction and maintenance studies, comparisons of primary and secondary endpoints between ustekinumab and placebo for the overall study population were performed using Cochran-MantelHaenszel chi-square test at a 2-sided significance level of 0.05 . To control the overall Type 1 error rate, the primary endpoint and major secondary endpoints were tested in a hierarchical order. Subsequent endpoints were considered statistically significant only if the preceding endpoint in the hierarchy was positive. Patients with treatment failure (i.e., CD-related surgeries, prohibited changes to concomitant CD medications, initiation of prohibited medications), or insufficient data to calculate CDAI scores (i.e., $<4$ of 8 CDAI components available) were considered unresponsive or not in remission. For maintenance, patients who had a loss of response or discontinued study agent due to lack of therapeutic effect or an adverse event of worsening disease were also considered as treatment failures. Treatment-failure and missing data rules were also applied for dichotomous secondary endpoints. For continuous endpoints, baseline values (from induction week 0 ) were assigned from time of the treatment failure, and the last available observation was carried forward for missing data. The sample size for Japanese subpopulation was determined with reference to the guidance from Japanese Ministry of Health, Labour and Welfare $^{20,21}$ The results of the Japanese subpopulation analysis were summarized descriptively, and consistency in efficacy and safety outcomes between the Japanese subpopulation and overall population were evaluated.

\section{RESULTS}

\section{Patient Disposition}

In UNITI-1, a total of 741 patients were randomized, of which 56 were from Japan (placebo, 18; ustekinumab $130 \mathrm{mg}$, 19; ustekinumab $\sim 6 \mathrm{mg} / \mathrm{kg}, 19$ ). In UNITI-2, of the 628 patients randomized, 26 were from Japan (placebo, 9; ustekinumab $130 \mathrm{mg}$, 8; ustekinumab $\sim 6 \mathrm{mg} / \mathrm{kg}$, 9). A total of 397 patients, including 21 patients from Japan, responded to ustekinumab treatment in induction studies and were enrolled in IM-UNITI as the primary (randomized) population (placebo, 4; ustekinumab $90 \mathrm{mg}$ SC q8w group, 9; ustekinumab $90 \mathrm{mg}$ SC q12w group, 8). A total of 884 (Japan 53) patients who were nonresponders to ustekinumab or placebo or who were responders to placebo entered IMUNITI as a nonrandomized population (Fig. 1). The demographic characteristics and baseline disease characteristics in the Japanese subpopulation were similar to those of the overall study population, except for median body weight, which was lesser, and CRP level, which was greater in the Japanese subpopulation than the overall study population (Table 1).

\section{Efficacy in Induction Studies}

\section{1) Primary Efficacy Endpoint}

In UNITI-1, the proportion of patients achieving clinical response at week 6 was greater in ustekinumab groups (130 mg: Japanese subgroup, 36.8\% [7/19]; overall, 34.3\%; 6 mg/ kg: Japanese subgroup, 31.6\% [6/19]; overall, 33.7\%) than in the placebo group (Japanese subgroup, 21.5\% [5/18]; overall, 27.8\%) (Fig. 2A). Similarly, in UNITI-2, the proportion of patients achieving clinical response at week 6 was greater in ustekinumab groups (130 mg: Japanese subgroup, 37.5\% [3/8]; overall, $51.7 \%$; $6 \mathrm{mg} / \mathrm{kg}$ : Japanese subgroup, $55.6 \%$ [5/9]; overall, 55.5\%) than in the placebo group (Japanese subgroup, 11.1\% [1/9]; overall, 28.7\%) (Fig. 2B) 
Table 1. Patient Demographics and Clinical Characteristics of the Overall Study Population and Japanese Subpopulation: Induction Studies and Maintenance Study

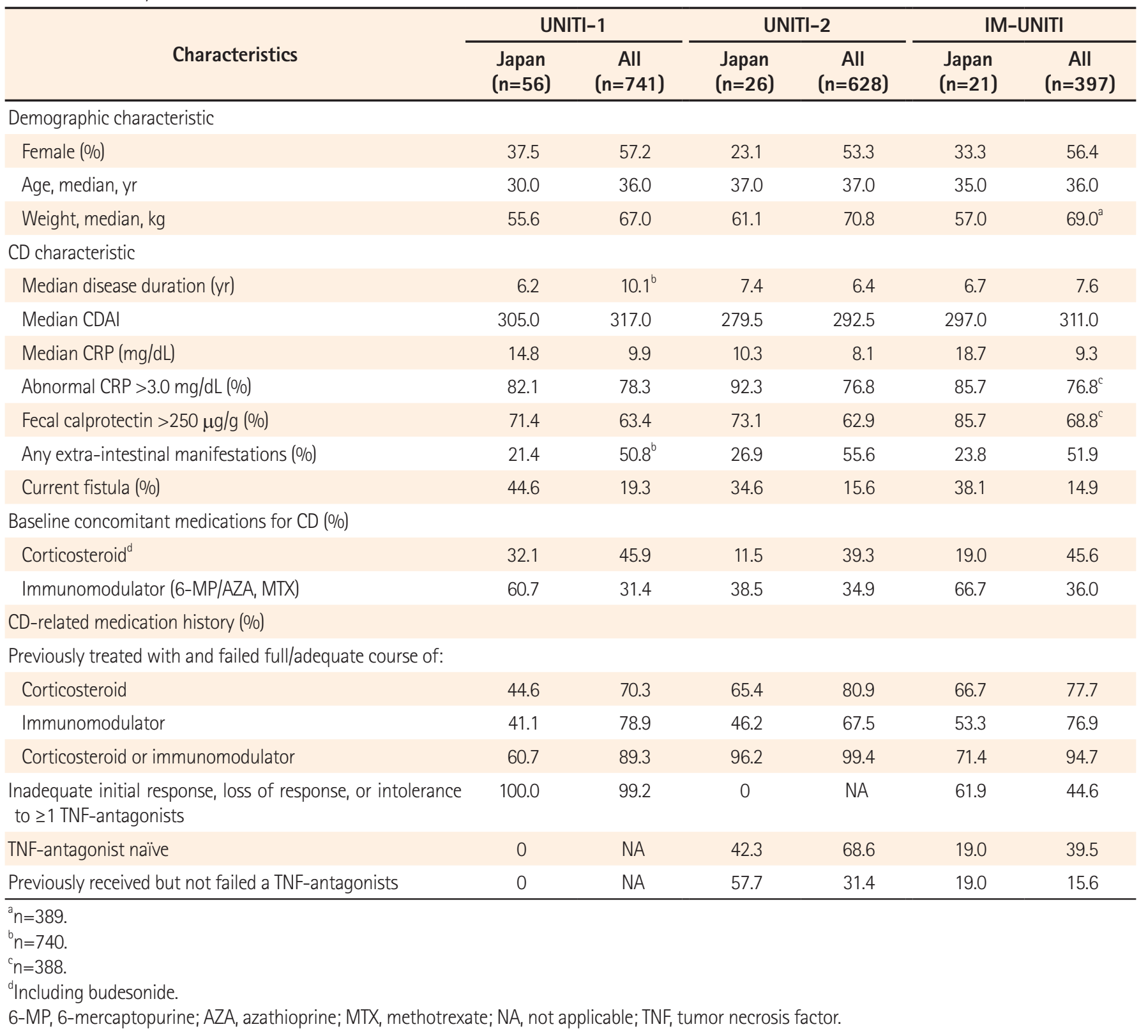

\section{2) Major Secondary Efficacy Endpoints}

In both the induction studies, a greater proportion of patients treated with ustekinumab (both $130 \mathrm{mg}$ and $\sim 6 \mathrm{mg} /$ $\mathrm{kg}$ ) achieved clinical response and remission at week 8 than the placebo group (in Japanese subpopulation and overall study population) (Table 2). A higher proportion of patients attained the 70-point response at week 3 or week 6 with either of the ustekinumab induction doses than those in the placebo group, and the response was sustained at week 6 in both the Japanese and overall study populations (Table 2).
Thus, the primary and major secondary endpoints were met and both induction doses were better than the placebo in the subset of Japanese patients, and results were generally similar to the overall population. ${ }^{19}$

\section{3) Other Efficacy Endpoints}

The median change in CDAI scores in the Japanese subpopulation was generally better in both ustekinumab induction groups when compared with the placebo (Fig. 3AD). In the Japanese subpopulation, the median serum CRP 

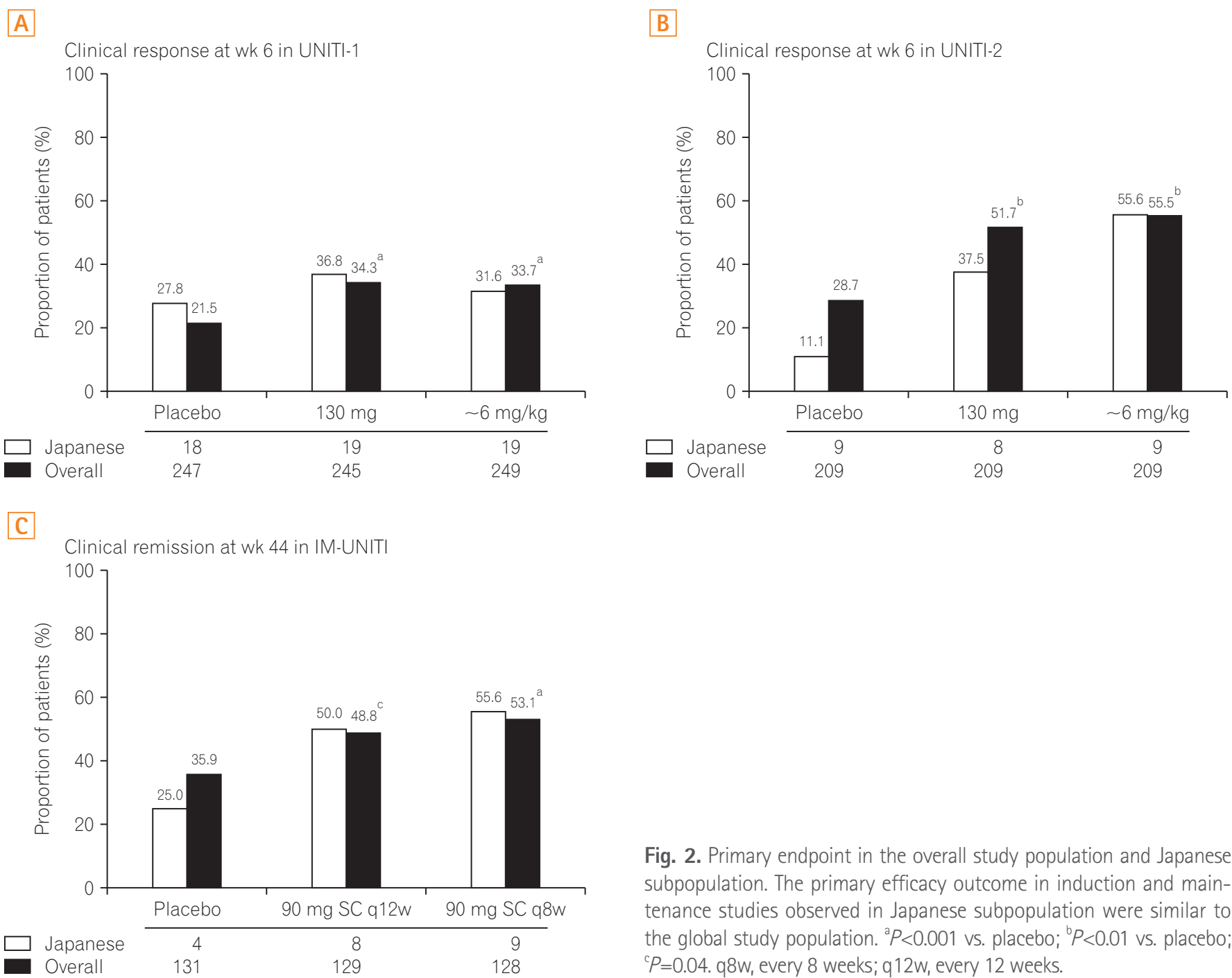

Fig. 2. Primary endpoint in the overall study population and Japanese subpopulation. The primary efficacy outcome in induction and maintenance studies observed in Japanese subpopulation were similar to the global study population. ${ }^{a} P<0.001$ vs. placebo; ${ }^{b} P<0.01$ vs. placebo; ${ }^{c} P=0.04$. q8 $w_{1}$ every 8 weeks; $q 12 w_{1}$ every 12 weeks.

Table 2. Major Secondary Endpoints of Induction Studies in the Overall Population and Japanese Subpopulation

\begin{tabular}{|c|c|c|c|c|c|c|c|c|c|c|c|c|}
\hline \multirow{3}{*}{ Endpoints } & \multicolumn{6}{|c|}{ UNITI-1 } & \multicolumn{6}{|c|}{ UNITI-2 } \\
\hline & \multicolumn{2}{|c|}{ Placebo } & \multicolumn{2}{|c|}{$130 \mathrm{mg}$} & \multicolumn{2}{|c|}{$\sim 6 \mathrm{mg} / \mathrm{kg}$} & \multicolumn{2}{|c|}{ Placebo } & \multicolumn{2}{|c|}{$130 \mathrm{mg}$} & \multicolumn{2}{|c|}{$\sim 6 \mathrm{mg} / \mathrm{kg}$} \\
\hline & Japan & All & Japan & All & Japan & All & Japan & All & Japan & All & Japan & All \\
\hline Number & 18 & 247 & 19 & 245 & 19 & 249 & 9 & 209 & 8 & 209 & 9 & 209 \\
\hline Clinical response at wk 8 & 27.8 & 20.2 & 21.1 & $33.5^{\mathrm{a}}$ & 52.6 & $37.8^{b}$ & 0 & 32.1 & 37.5 & $47.4^{b}$ & 66.7 & $57.9^{b}$ \\
\hline Clinical remission at wk 8 & 5.6 & 7.3 & 10.3 & $15.9^{\mathrm{a}}$ & 26.3 & $20.9^{b}$ & 0 & 19.6 & 25.0 & $30.6^{a}$ & 22.2 & $40.2^{b}$ \\
\hline 70-Point response at wk 3 & 27.8 & 27.1 & 42.1 & $38.4^{\mathrm{a}}$ & 42.1 & $40.6^{a}$ & 11.1 & 31.6 & 25.0 & $49.3^{b}$ & 44.4 & $50.7^{b}$ \\
\hline 70-Point response at wk 6 & 44.4 & 30.4 & 42.1 & $46.1^{b}$ & 52.6 & $43.8^{\mathrm{a}}$ & 11.1 & 38.8 & 50.0 & $58.9^{b}$ & 77.8 & $64.6^{b}$ \\
\hline
\end{tabular}

Values are presented as percentage.

${ }^{\mathrm{a}} P<0.01$ vs. placebo.

${ }^{\mathrm{b}} P<0.001$ vs. placebo.

concentration was considerably reduced at the earliest timepoint (week 3 ) in both the ustekinumab induction groups (130 mg: UNITI-1, -2.3 mg/L; UNITI-2, -1.78 mg/L; 6 mg/ kg: UNITI-1, -3.20 mg/L; UNITI-2, -7.20 mg/L) compared with the corresponding placebo groups (UNITI-1, $2.17 \mathrm{mg} / \mathrm{L}$; UNITI-2, $0.55 \mathrm{mg} / \mathrm{L}$ ). The reductions seen after ustekinumab 
A

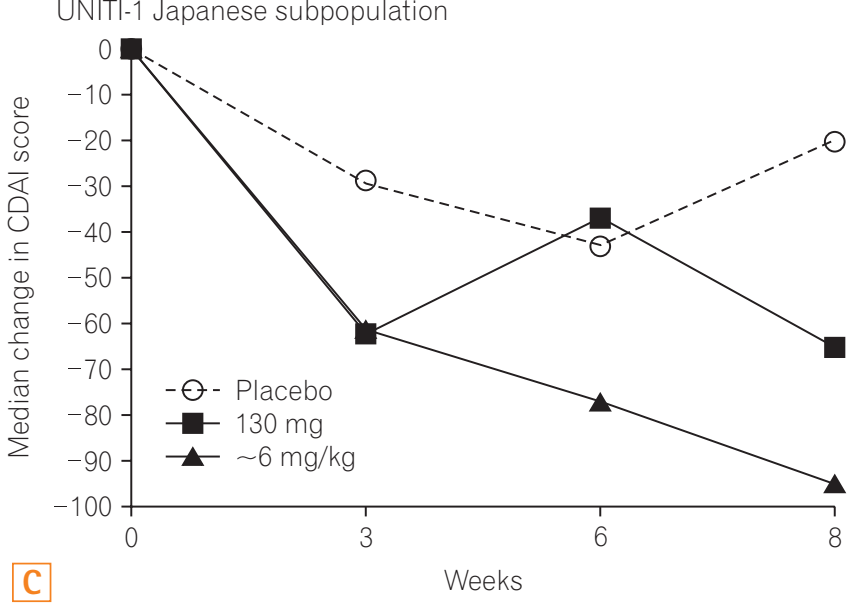

C

UNITI-2 Japanese subpopulation

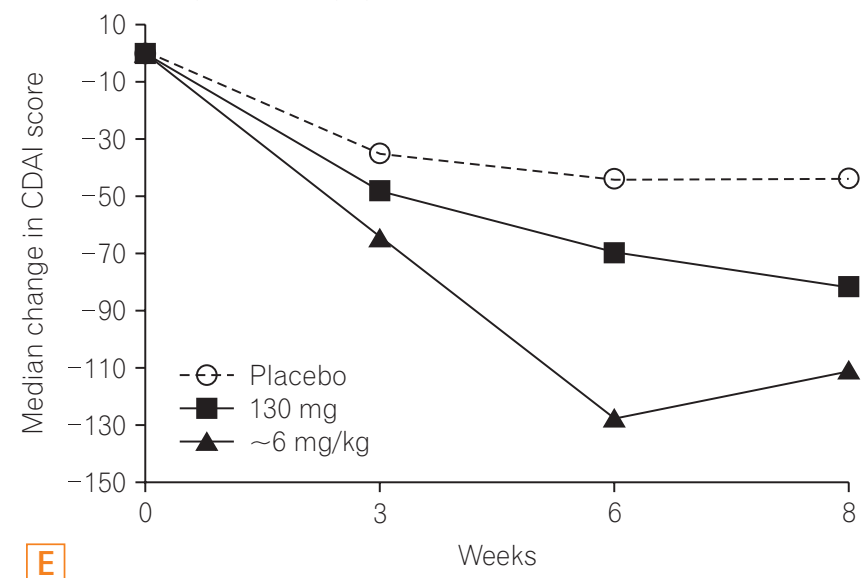

E

IM-UNITI Japanese subpopulation

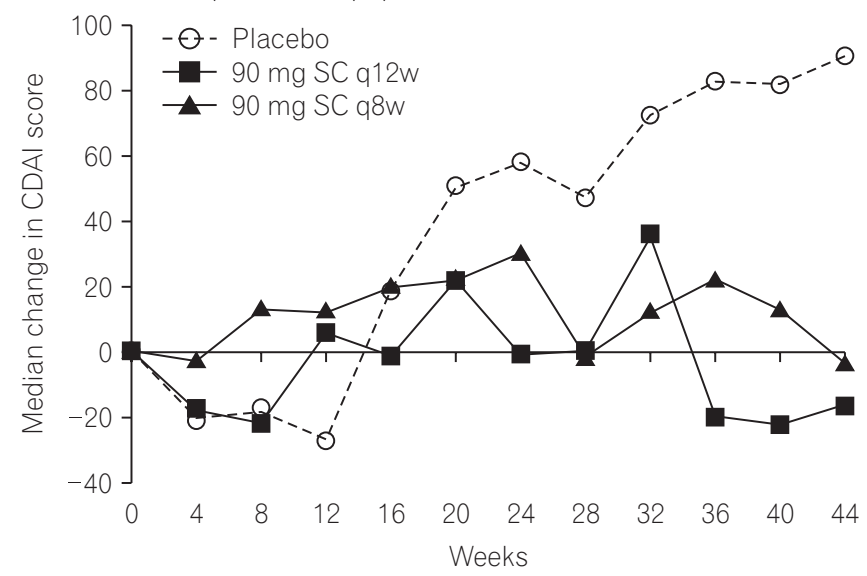

B

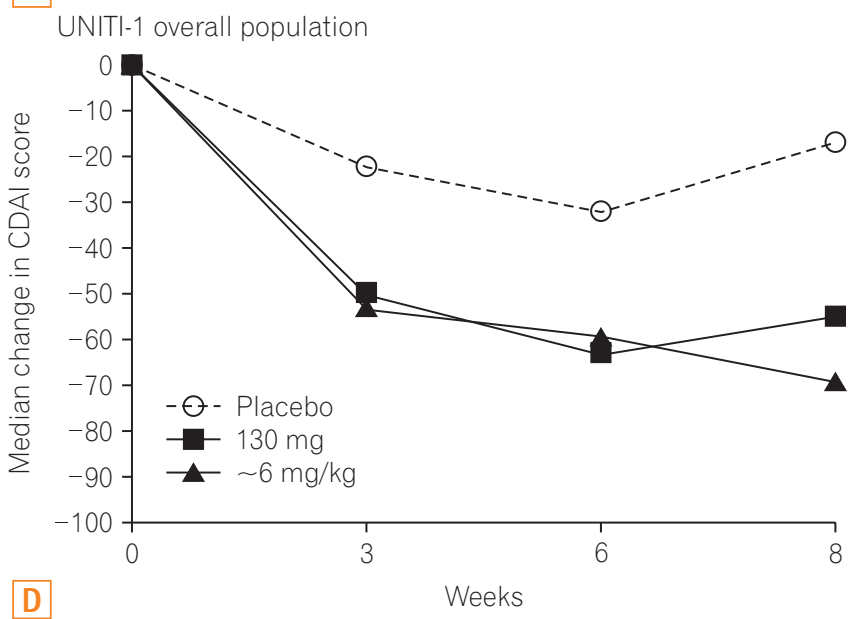

UNITI-2 overall population
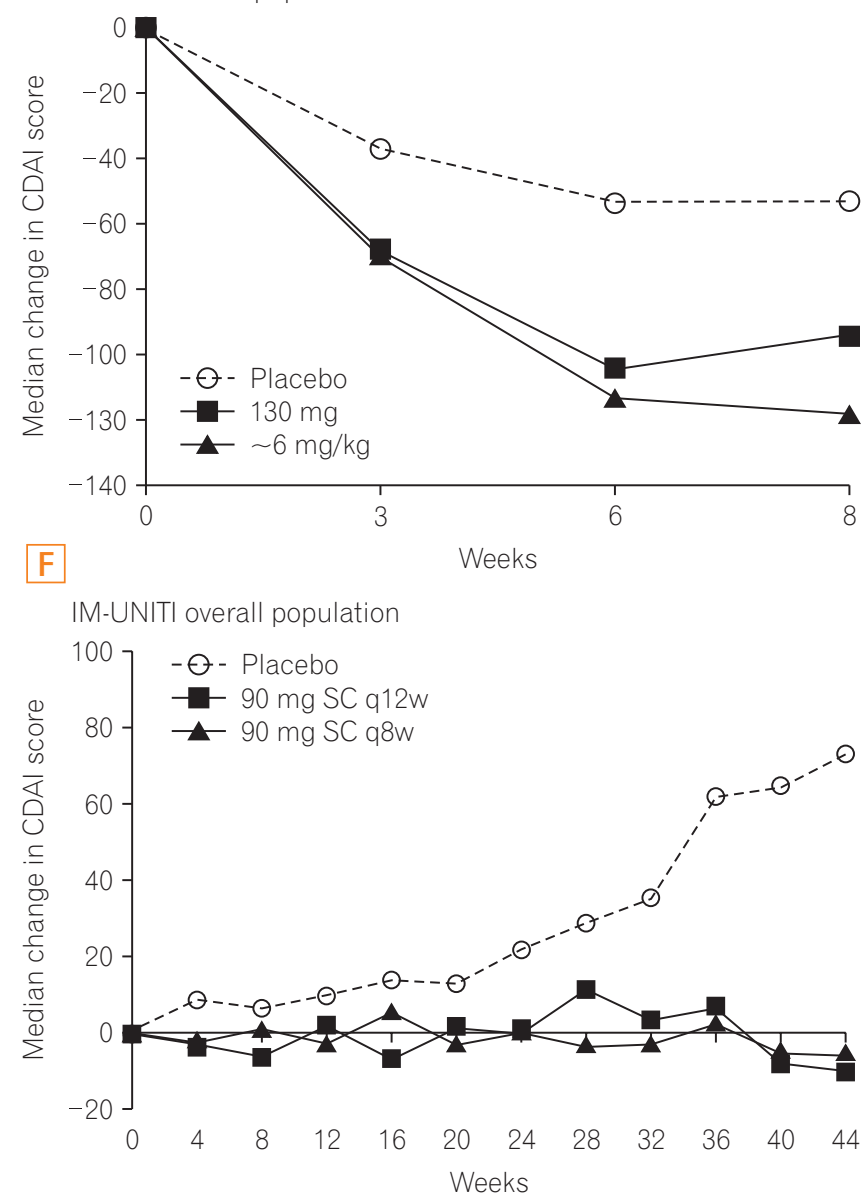

Fig. 3. Median change in CDAl scores over time. In the induction studies and maintenance study, the median change in CDAl scores consistently improved with ustekinumab compared with that with placebo in the Japanese subpopulation and overall study population. All $P<0.05$ vs. placebo in the overall population of the UNITI-1, UNITI-2, and IM-UNITI studies. $912 \mathrm{w}_{\text {, every }} 12$ weeks; q8w, every 8 weeks. Panel B, D, and F are adapted from Feagan BG, et al. N Engl J Med 2016;375:1946-1960, with permission Massachusetts Medical Society. ${ }^{19}$

treatment were persistent through week 8 . These reductions in CRP concentration were also similar to those in the overall population (Supplementary Fig. 1).
In the Japanese subpopulation of UNITI-1, the median fecal calprotectin concentration at week 6 was reduced $(-6.5$ $\mathrm{mg} / \mathrm{kg}$ ) in the ustekinumab $130 \mathrm{mg}$ group, while it was in- 
creased by $2.11 \mathrm{mg} / \mathrm{kg}$ in the ustekinumab $\sim 6 \mathrm{mg} / \mathrm{kg}$ group and by $15.84 \mathrm{mg} / \mathrm{kg}$ in the placebo group. In the UNITI-2 Japanese subpopulation, there was no change in the fecal calprotectin concentration at week 6 in the ustekinumab $130 \mathrm{mg}$ group; however, it was reduced $(-108.23 \mathrm{mg} / \mathrm{kg})$ in the ustekinumab $\sim 6 \mathrm{mg} / \mathrm{kg}$ group and was increased by 2.97 $\mathrm{mg} / \mathrm{kg}$ in the placebo group. In the overall study population, significantly greater reductions in fecal calprotectin were observed at week 6 in both the ustekinumab induction groups compared with the placebo group $(P<0.001)$ (Supplementary Fig. 2A).

\section{Efficacy in Maintenance Study}

\section{1) Primary Efficacy Endpoint}

The proportion of responders to ustekinumab induction who exhibited clinical remission at week 44 during maintenance was greater in the ustekinumab groups (90 mg SC q12w: overall, 48.8\%; Japanese subgroup, 50.0\% [4/8]; $90 \mathrm{mg}$ SC q8w: overall, 53.1\%; Japanese subgroup, 55.6\% [5/9]) than in the placebo group (overall, $35.9 \%$; Japanese subgroup, $25.0 \%[1 / 4]$ ) (Fig. 2C).

\section{2) Major Secondary Efficacy Endpoints}

Similar to the results observed for the overall study population, a higher proportion of patients in the Japanese subgroup showed sustained clinical remission (remission at weeks 36,40 , and 44 ) in the ustekinumab groups (90 $\mathrm{mg}$ SC: $\mathrm{q} 12 \mathrm{~W}, 37.5 \%$ [3/8]; q8w, 55.6\% [5/9]) than in the placebo group (25.0\% [1/4]) (Supplementary Fig. 3).

The proportion of Japanese patients remaining in remission at week 44 among those in remission after induction, at maintenance baseline (remission in remitters), was $80 \%$ $(4 / 5)$ and 50\% (2/4) in the ustekinumab $90 \mathrm{mg}$ SC q8w and q12w groups, respectively, while $1 / 1$ patient maintained remission on SC placebo (i.e., the only patient in remission, when randomized to SC placebo maintenance, stayed in remission at week 44). In the overall study population, a greater proportion of patients who were in remission at maintenance baseline maintained remission with ustekinumab $90 \mathrm{mg}$ SC q8w treatment (67\%) than those with q12w (56\%) and placebo (46\%) treatments. Number of patients in the Japanese subgroup achieving corticosteroid-free remission at week 44 were greater with ustekinumab maintenance (90 mg SC: q12w, 55.6\% [5/9]; q8w, 50\% [4/8]) than with placebo (25\% $[1 / 4])$. In the overall study population, the proportion of patients in corticosteroid-free remission at week 44 was significantly greater in the $\mathrm{q} 8 \mathrm{w}$ group $(P=0.004)$ and nominally significant in the $\mathrm{q} 12 \mathrm{w}$ group $(P=0.035$; as per hierarchical testing procedure) than with placebo (Supplementary Fig. 4).

In the Japanese subpopulation, clinical remission at week 44 among those who were refractory or intolerant to TNF antagonist therapy (subset of patients from UNITI-1) was achieved with $90 \mathrm{mg} \mathrm{SC} \mathrm{q12w} \mathrm{(33.3 \%} \mathrm{[2/6])} \mathrm{and} \mathrm{q8w}$ ustekinumab treatment $(50.0 \%[2 / 4])$, but not with placebo treatment $(0 \%[0 / 3])$. Similarly, in the subset of UNITI-1 patients from the overall population, a greater proportion of patients receiving ustekinumab $90 \mathrm{mg}$ SC q12w (38.6\%) and q8w (41.1\%) maintained clinical remission at week 44 than those on placebo (26.2\%) (Supplementary Fig. 5).

\section{3) Other Efficacy Endpoints}

Throughout the maintenance study, the median change in CDAI scores was generally improved in both the ustekinumab dose groups and was better than in the placebo group for both the Japanese subpopulation and the overall study population (Fig. $3 \mathrm{E}$ and F).

A normal CRP level at week 44 was achieved in Japanese patients who had baseline CRP $>3 \mathrm{mg} / \mathrm{L}$ in the induction studies, when treated with ustekinumab $90 \mathrm{mg}$ SC q12w (42.9\% [3/7]), while normal levels were not achieved with $90 \mathrm{mg}$ SC q8w (0/8 patients) or placebo (0/3 patients) treatment. However, in the overall population, proportion of patients attaining a normalized CRP was greater for both the ustekinumab regimens than the placebo. The median change in fecal calprotectin concentration at week 44 in the Japanese subpopulation was considerably reduced with both $90 \mathrm{mg} \mathrm{SC} \mathrm{q12w}(-102.3 \mathrm{mg} / \mathrm{kg})$ and q8w treatment $(-17.90 \mathrm{mg} / \mathrm{kg})$, while it increased over the maintenance period in the SC placebo group $(168.15 \mathrm{mg} / \mathrm{kg})$. In the overall study population, there was no change in fecal calprotectin levels at week 44 in both the ustekinumab groups, while it increased in the placebo group $(153.85 \mathrm{mg} / \mathrm{kg}$ ) (Supplementary Fig. 2B).

In the Japanese subpopulation, when nonrandomized patients (nonresponders to ustekinumab IV induction, $\mathrm{n}=31$; Fig. 1) of the maintenance study were treated with an additional SC dose of ustekinumab $(90 \mathrm{mg})$ at week 0 of maintenance, $61.3 \%(n=19)$ achieved clinical response 8 weeks later. A total of 22 of these nonrandomized patients continued ustekinumab, receiving $90 \mathrm{mg}$ q8w maintenance regimen. Of these patients, $54.5 \%(n=12)$ maintained clinical response and $36.4 \%(\mathrm{n}=8)$ achieved clinical remission at week 44 . In the overall study population, $50.5 \%$ of nonrandomized patients achieved clinical response at week 8 (after ustekinumab $90 \mathrm{mg}$ SC injection at week 0) and $68.1 \%$ of 
those patients continued to maintain a clinical response and $50.2 \%$ were in remission at week 44 .

\section{Safety}

In the combined Japanese subpopulations of both induction studies, TEAEs were reported in $44.4 \%$ of patients receiving ustekinumab ( $130 \mathrm{mg}$ ), $50.0 \%$ in the $\sim 6 \mathrm{mg} / \mathrm{kg}$ group, and $55.6 \%$ in the placebo group. A consistent pattern was observed in the overall study population (ustekinumab 130 mg, 58.4\%; 6 mg, 60.4\%; placebo, 60.5\%) (Table 3). The most frequently reported TEAEs ( $>2$ patients in ustekinumab groups) in the Japanese subpopulation of induction studies were nasopharyngitis $(n=6,10.9 \%)$ and pyrexia $(n=3,5.5 \%)$. Similarly, in the overall population, TEAEs of nasopharyngitis $(4.6 \%)$ and pyrexia (5.9\%) were commonly reported. The incidence of infection TEAEs was similar across the treat- ment groups in Japanese patients (ustekinumab $130 \mathrm{mg}$, $18.5 \% ; \sim 6 \mathrm{mg} / \mathrm{kg}, 25.0 \%$; placebo, $22.2 \%$ ) and none of these were serious infections. The proportion of overall serious TEAEs in the induction studies was also not higher following ustekinumab treatment and occurred at similar frequencies in the Japanese subpopulation (both ustekinumab doses, $3.6 \%$; placebo, $7.4 \%$ ) and the overall study population (both ustekinumab doses, $5.1 \%$; placebo, $6.0 \%$ ). One patient in the placebo group experienced a TEAE leading to study drug discontinuation (Table 3).

In the IM-UNITI maintenance study, TEAEs occurred at similar frequencies across the treatment groups in both the Japanese subpopulation and the overall study population (Table 4). The most frequently reported TEAEs ( $>2$ patients in ustekinumab groups) in the Japanese subpopulation were nasopharyngitis $(n=6,35.3 \%)$ and headache $(n=4,23.5 \%)$. The other infections observed in $\geq 2$ Japanese patients in

Table 3. Summary of Safety Results through Week 8 (Combined UNITI-1 and-2 Study)

\begin{tabular}{|c|c|c|c|c|c|c|c|c|}
\hline \multirow{2}{*}{ Description } & \multicolumn{2}{|c|}{ Placebo } & \multicolumn{2}{|c|}{$\begin{array}{c}\text { Ustekinumab } \\
130 \mathrm{mg}\end{array}$} & \multicolumn{2}{|c|}{$\begin{array}{l}\text { Ustekinumab } \\
\sim 6 \mathrm{mg} / \mathrm{kg}\end{array}$} & \multicolumn{2}{|c|}{ Combined } \\
\hline & $\begin{array}{l}\text { Japan } \\
(n=27)\end{array}$ & $\begin{array}{c}\text { All } \\
(n=466)\end{array}$ & $\begin{array}{l}\text { Japan } \\
(n=28)\end{array}$ & $\begin{array}{c}\text { All } \\
(n=471)\end{array}$ & $\begin{array}{l}\text { Japan } \\
(n=28)\end{array}$ & $\begin{array}{c}\text { All } \\
(n=470)\end{array}$ & $\begin{array}{l}\text { Japan } \\
(n=55)\end{array}$ & $\begin{array}{c}\text { All } \\
(n=941)\end{array}$ \\
\hline Average duration of follow-up (wk) & 7.80 & 8.18 & 7.93 & 8.22 & 8.10 & 8.16 & 8.02 & 8.19 \\
\hline TEAE & $15(55.6)$ & $282(60.5)$ & $12(44.4)$ & $275(58.4)$ & $14(50.0)$ & $284(60.4)$ & $26(47.3)$ & $559(59.4)$ \\
\hline Serious TEAE & $2(7.4)$ & $28(6.0)$ & 0 & $23(4.9)$ & $2(7.1)$ & $25(5.3)$ & $2(3.6)$ & $48(5.1)$ \\
\hline Infection $^{a}$ & $6(22.2)$ & $108(23.2)$ & $5(18.5)$ & $92(19.5)$ & $7(25.0)$ & $111(23.6)$ & $12(21.8)$ & $203(21.6)$ \\
\hline Serious infection ${ }^{a}$ & 0 & $6(1.3)$ & 0 & $7(1.5)$ & 0 & $8(1.7)$ & 0 & $15(1.6)$ \\
\hline TEAEs leading to discontinuation of study drug & $1(3.7)$ & $19(4.1)$ & 0 & $8(1.7)$ & 0 & $8(1.7)$ & 0 & $16(1.7)$ \\
\hline
\end{tabular}

Values are presented as number (\%).

anfection as assessed by the investigator.

TEAE, treatment-emergent adverse event.

Table 4. Summary of Safety Results through Week 44 of the IM-UNITI Study

\begin{tabular}{|c|c|c|c|c|c|c|c|c|}
\hline \multirow[b]{2}{*}{ Description } & \multicolumn{2}{|c|}{ Placebo } & \multicolumn{2}{|c|}{$90 \mathrm{mg} \mathrm{SC} \mathrm{q12w}$} & \multicolumn{2}{|c|}{$90 \mathrm{mg} \mathrm{SC}$ q8w } & \multicolumn{2}{|c|}{ Combined } \\
\hline & $\begin{array}{l}\text { Japan } \\
(n=4)\end{array}$ & $\begin{array}{c}\text { All } \\
(n=133)\end{array}$ & $\begin{array}{l}\text { Japan } \\
(n=8)\end{array}$ & $\begin{array}{c}\text { All } \\
(n=132)\end{array}$ & $\begin{array}{l}\text { Japan } \\
(n=9)\end{array}$ & $\begin{array}{c}\text { All } \\
(n=131)\end{array}$ & $\begin{array}{l}\text { Japan } \\
(n=17)\end{array}$ & $\begin{array}{c}\text { All } \\
(n=263)\end{array}$ \\
\hline Average duration of follow-up (wk) & 30.2 & 32.0 & 39.3 & 36.6 & 36.0 & 35.2 & 37.6 & 35.9 \\
\hline TEAE & $4(100.0)$ & $111(83.5)$ & $8(100.0)$ & $106(80.3)$ & $8(88.9)$ & $107(81.7)$ & $16(94.1)$ & $213(81.0)$ \\
\hline Serious TEAE & 0 & $20(15.0)$ & $2(25.0)$ & $16(12.1)$ & $1(11.1)$ & $13(9.9)$ & $3(17.6)$ & $29(11.0)$ \\
\hline Infection $^{\mathrm{a}}$ & $2(50.0)$ & $66(49.6)$ & $5(62.5)$ & $61(46.2)$ & 8 (88.9) & $63(48.1)$ & $13(76.5)$ & $124(47.1)$ \\
\hline Serious infection $^{a}$ & 0 & $3(2.3)$ & $2(25.0)$ & $7(5.3)$ & 0 & $3(2.3)$ & $2(11.8)$ & $10(3.8)$ \\
\hline TEAEs leading to discontinuation of study drug & 0 & $8(6.0)$ & $1(12.5)$ & $10(7.6)$ & 0 & $4(3.1)$ & $1(5.9)$ & $14(5.3)$ \\
\hline
\end{tabular}

Values are presented as number (\%).

anfection as assessed by the investigator

q12w, every 12 weeks; $q 8 \mathrm{w}$, every 8 weeks; TEAE, treatment-emergent adverse event. 
maintenance were anal abscess, pharyngitis, and vulvovaginal candidiasis $(\mathrm{n}=2,11.8 \%$; each), and this was similar to the values for the overall study populations. A total of 3 serious TEAEs ( $\mathrm{q} 12 \mathrm{w}, \mathrm{n}=2$; $\mathrm{q} 8 \mathrm{w}, \mathrm{n}=1$ ) were observed in the ustekinumab group within the Japanese subpopulation. Two of these were serious infections (anal abscess and bacteremia) observed in the $90 \mathrm{mg} \mathrm{SC} \mathrm{q12w}$ group. The serious TEAE of anal abscess was the only adverse event leading to discontinuation of the study agent (Table 4).

There were no deaths, malignancies, events of tuberculosis, or opportunistic infections reported in the Japanese subpopulation through 52 weeks of therapy. None of the Japanese patients in the ustekinumab group reported any TEAEs related to infusions in the induction or maintenance studies.

\section{Pharmacokinetics and Immunogenicity}

No clear difference in serum ustekinumab concentrations was observed between Japanese patients and non-Japanese patients in the induction studies or the maintenance study, regardless of the dose received (Supplementary Figs 6 and 7). In the overall population, 2 patients who received ustekinumab (130 mg) IV developed antibodies to ustekinumab in the induction studies and 27 patients (2.3\%) developed antibodies in the maintenance study, determined using a drug tolerant assay. None of the Japanese patients who received ustekinumab developed antibodies to ustekinumab in the induction studies and 2 Japanese patients (9.5\%) developed antibodies in the maintenance study.

\section{DISCUSSION}

Genetic variations that may increase predisposition to $\mathrm{CD}$ or affect treatment responses among ethnic populations have been reported. For example, there are certain coding variants in nucleotide-binding oligomerization domaincontaining protein 2 (NOD2) that have a large effect on IBD risk in Caucasians; however, such variants in allele frequency in Asian populations, including Japanese population, is absent, which suggests that there is genetic heterogeneity in predisposition to $\mathrm{CD}^{22,23}$ Furthermore, the treatment effect of thiopurine may differ between Asians and Caucasians because of the prevalence of a variant in NUDT15, which is associated with thiopurine-induced leukopenia in patients with IBD. ${ }^{24}$ Because differences in IBD treatment responses among ethnic populations are possible due to genetic het- erogeneity, ${ }^{24}$ it is important to assess the consistency or difference of treatment outcomes between specific ethnic populations and overall population.

The phase 3 global clinical study program of ustekinumab studied efficacy and safety in a wide range of patients with moderately to severely active CD who had been previously treated with TNF-antagonists or conventional therapy. ${ }^{19}$ In the Japanese subpopulation of this phase 3 program, ustekinumab $130 \mathrm{mg}$ and $\sim 6 \mathrm{mg} / \mathrm{kg}$ IV induction therapy showed increased clinical response rates at week 6 over placebo, both among patients who were refractory to TNFantagonists as well as to conventional therapy. Among patients who responded to IV ustekinumab in the induction studies, treatment with SC ustekinumab (90 mg q12w and q8w) maintenance therapy demonstrated increased rates of clinical remission and response at week 44 .

The primary and major secondary efficacy outcomes in induction and maintenance studies observed in the Japanese subpopulation were consistent with the findings observed in the overall global study population. ${ }^{19}$ In both induction studies, $\sim 6 \mathrm{mg} / \mathrm{kg}$ ustekinumab showed higher rates of clinical response and remission than $130 \mathrm{mg}$ dose, particularly at week 8 . Patients in the UNITI-2 study who had failed responses only to conventional therapy achieved higher rates of response and remission than the patients with failed responses to TNF-antagonist in the UNITI-1 study, possibly because these patients had a less refractory disease. Similar to the overall study population, in the Japanese subpopulation treated with ustekinumab, induction demonstrated greater reductions in CRP concentration than the placebo even at the first post-baseline visit at week 3 . Reductions in fecal calprotectin levels were observed only with $130 \mathrm{mg}$ ustekinumab treatment in UNITI-1 study and $\sim 6 \mathrm{mg}$ / $\mathrm{kg}$ ustekinumab treatment in UNITI-2 study in the Japanese subpopulation; whereas in the overall study population, both doses of ustekinumab showed reductions in fecal calprotectin and $\sim 6 \mathrm{mg}$ ustekinumab dose showed the highest reduction in both studies. This may be due to high variability in calprotectin levels and limited number of Japanese patients. However, overall treatment with ustekinumab improved objective markers of inflammation along with improvements in clinical response and remission in the Japanese subpopulation similar to the overall study population.

In the maintenance study, in Japanese patients who responded to IV ustekinumab induction, treatment with SC ustekinumab (90 mg q12w and q8w) maintained clinical remission, clinical response, remission among those in remission after induction, and corticosteroid-free remis- 
sion as well as sustained remission. Although both doses of SC ustekinumab (90 mg q12w and q8w) demonstrated improvement in clinical outcomes, q8w dose showed the highest improvement in both the Japanese and overall populations. In Japanese patients who had elevated CRP concentration $(>3 \mathrm{mg} / \mathrm{L})$ at baseline of induction studies, treatment with ustekinumab $90 \mathrm{mg}$ SC q12w normalized CRP levels at week 44. A considerable reduction in fecal calprotectin levels at week 44 was also observed with ustekinumab maintenance regimens in the Japanese subpopulation. Japanese patients who were nonresponders to IV ustekinumab during induction (nonrandomized patients) also showed greater response during the additional maintenance ustekinumab SC treatment, which corroborated the results of the overall study population as well as results in the randomized primary population.

Ustekinumab IV induction and SC maintenance regimens were well tolerated in the Japanese subpopulation and in the overall study population. The rates of TEAEs and serious TEAEs were similar across the treatment groups. There were no reports of serious infections with ustekinumab IV treatment during induction in the Japanese subpopulation; whereas only 2 patients reported serious infections with ustekinumab $90 \mathrm{mg}$ SC q12w during maintenance. Treatment with ustekinumab was associated with only one TEAE leading to treatment discontinuation. Additionally, there were no reports of infusion reactions, and no deaths were reported in these studies through week 44, in either the Japanese or overall populations. Overall, ustekinumab treatment showed no safety concerns and results were consistent with those for the overall study population as well as for those with the previous studies conducted in CD patients ${ }^{18,19}$ and with previous long-term studies conducted for other indications, including psoriasis and psoriatic arthritis. ${ }^{17,25-27}$ No clear difference in serum ustekinumab concentrations was observed between the Japanese patients and non-Japanese patients in induction studies or the maintenance study.

The sample size in this Japanese subpopulation analysis was small to perform statistical analysis; however, efficacy and safety outcomes in Japanese patients were analogous to the results of global population.

To the best of our knowledge, this is the first global IBD study to demonstrate findings of the Japanese subpopulation consistent to that of the overall study population. The IV ustekinumab as an induction therapy was more effective than placebo in Japanese patients with moderate to severely active $\mathrm{CD}$, who were refractory to either TNF antagonists or conventional therapy. Among the responders to IV ustekinumab induction, treatment with SC ustekinumab was also more effective than placebo in maintaining clinical response and remission along with other efficacy outcomes. Both ustekinumab induction and maintenance regimens were generally well tolerated. Despite genetic variations that may exist in the Japanese population, these data suggest that ustekinumab provides efficacy in Japanese patients with CD, similar to observations in the global population. Ustekinumab treatment exhibits a favorable benefit-risk balance for CD; therefore, it could be considered as a new therapeutic option for moderate to severely active CD in Japanese patients who have failed conventional therapies or have additionally previously failed TNF antagonists.

\section{ACKNOWLEDGEMENTS}

The authors thank the study participants without whom this study would never have been accomplished and also thank the investigators for their participation in the study. The authors also thank Dr. Takayuki Ota for his contribution to this manuscript. Writing assistance was provided by Ramji Narayanan, ISMPP CMPP ${ }^{\mathrm{TM}}$ (SIRO Clinpharm Pvt. Ltd., Thane, India), funded by Janssen Pharmaceutical K.K., Japan and publication support was provided by Kenichiro Tsutsumi (Janssen Pharmaceutical K.K., Tokyo, Japan). This study was funded by Janssen Research \& Development, LLC and Janssen Pharmaceutical K.K., Japan.

\section{AUTHOR CONTRIBUTIONS}

T.H. and C.S. were involved in conception and design of the study. T.H. was also involved in acquisition of data. R.Z. was involved in analysis of data. All authors were involved in interpretation of data, drafting and critical revision of the manuscript and have approved the final manuscript for submission. All authors also had full access to all the data in the study and had final responsibility for the decision to submit for publication.

\section{REFERENCES}

1. Abraham C, Cho JH. Inflammatory bowel disease. N Engl J Med 2009;361:2066-2078.

2. Loftus EV Jr. Clinical epidemiology of inflammatory bowel disease: incidence, prevalence, and environmental influences. Gastroenterology 2004;126:1504-1517.

3. Ananthakrishnan AN. Epidemiology and risk factors for IBD. Nat Rev Gastroenterol Hepatol 2015;12:205-217. 
4. Asakura K, Nishiwaki Y, Inoue N, Hibi T, Watanabe M, Takebayashi T. Prevalence of ulcerative colitis and Crohn's disease in Japan. J Gastroenterol 2009;44:659-665.

5. Ng WK, Wong SH, Ng SC. Changing epidemiological trends of inflammatory bowel disease in Asia. Intest Res 2016;14:111119.

6. Hu PJ. Inflammatory bowel disease in Asia: the challenges and opportunities. Intest Res 2015;13:188-190.

7. Ng SC, Tang W, Leong RW, et al. Environmental risk factors in inflammatory bowel disease: a population-based case-control study in Asia-Pacific. Gut 2015;64:1063-1071.

8. Kanai T, Matsuoka K, Naganuma M, Hayashi A, Hisamatsu T. Diet, microbiota, and inflammatory bowel disease: lessons from Japanese foods. Korean J Intern Med 2014;29:409-415.

9. Amiot A, Peyrin-Biroulet L. Current, new and future biological agents on the horizon for the treatment of inflammatory bowel diseases. Therap Adv Gastroenterol 2015;8:66-82.

10. Tanida S, Ozeki K, Mizoshita T, et al. Managing refractory Crohn's disease: challenges and solutions. Clin Exp Gastroenterol 2015;8:131-140.

11. Hanauer SB, Feagan BG, Lichtenstein GR, et al. Maintenance infliximab for Crohn's disease: the ACCENT I randomised trial. Lancet 2002;359:1541-1549.

12. Hanauer SB, Sandborn WJ, Rutgeerts P, et al. Human anti-tumor necrosis factor monoclonal antibody (adalimumab) in Crohn's disease: the CLASSIC-I trial. Gastroenterology 2006;130:323333.

13. Colombel JF, Sandborn WJ, Rutgeerts P, et al. Adalimumab for maintenance of clinical response and remission in patients with Crohn's disease: the CHARM trial. Gastroenterology 2007;132:52-65

14. Sandborn WJ, Feagan BG, Stoinov S, et al. Certolizumab pegol for the treatment of Crohn's disease. N Engl J Med 2007;357:228-238.

15. Benson JM, Peritt D, Scallon BJ, et al. Discovery and mechanism of ustekinumab: a human monoclonal antibody targeting interleukin-12 and interleukin-23 for treatment of immunemediated disorders. MAbs 2011;3:535-545.

16. Leonardi CL, Kimball AB, Papp KA, et al. Efficacy and safety of ustekinumab, a human interleukin-12/23 monoclonal antibody, in patients with psoriasis: 76-week results from a randomised, double-blind, placebo-controlled trial (PHOENIX 1). Lancet 2008;371:1665-1674.
17. Igarashi A, Kato T, Kato M, Song M, Nakagawa H; Japanese Ustekinumab Study Group. Efficacy and safety of ustekinumab in Japanese patients with moderate-to-severe plaque-type psoriasis: long-term results from a phase $2 / 3$ clinical trial. J Dermatol 2012;39:242-252.

18. Sandborn WJ, Gasink C, Gao LL, et al. Ustekinumab induction and maintenance therapy in refractory Crohn's disease. N Engl J Med 2012;367:1519-1528.

19. Feagan BG, Sandborn WJ, Gasink C, et al. Ustekinumab as induction and maintenance therapy for Crohn's disease. N Engl J Med 2016;375:1946-1960.

20. Ministry of Health, Labour and Welfare. Basic principles on global clinical trials. Tokyo: Ministry of Health, Labour and Welfare, 2007.

21. Ministry of Health, Labour and Welfare. Basic principles on global clinical trials (reference cases). Tokyo: Ministry of Health, Labour and Welfare, 2012.

22. Liu JZ, van Sommeren S, Huang H, et al. Association analyses identify 38 susceptibility loci for inflammatory bowel disease and highlight shared genetic risk across populations. Nat Genet 2015;47:979-986.

23. Inoue N, Tamura K, Kinouchi Y, et al. Lack of common NOD2 variants in Japanese patients with Crohn's disease. Gastroenterology 2002;123:86-91.

24. Yang SK, Hong M, Baek J, et al. A common missense variant in NUDT15 confers susceptibility to thiopurine-induced leukopenia. Nat Genet 2014;46:1017-1020.

25. Papp KA, Griffiths CE, Gordon K, et al. Long-term safety of ustekinumab in patients with moderate-to-severe psoriasis: final results from 5 years of follow-up. Br J Dermatol 2013;168:844-854.

26. Gottlieb AB, Kalb RE, Langley RG, et al. Safety observations in 12095 patients with psoriasis enrolled in an international registry (PSOLAR): experience with infliximab and other systemic and biologic therapies. J Drugs Dermatol 2014;13:1441-1448.

27. Kavanaugh A, Puig L, Gottlieb AB, et al. Maintenance of clinical efficacy and radiographic benefit through two years of ustekinumab therapy in patients with active psoriatic arthritis: results from a randomized, placebo-controlled phase III trial. Arthritis Care Res (Hoboken) 2015;67:1739-1749. 
See "Efficacy and safety of ustekinumab in Japanese patients with moderately to severely active Crohn's disease: a subpopulation analysis of phase 3 induction and maintenance studies" on page 475-486.

A

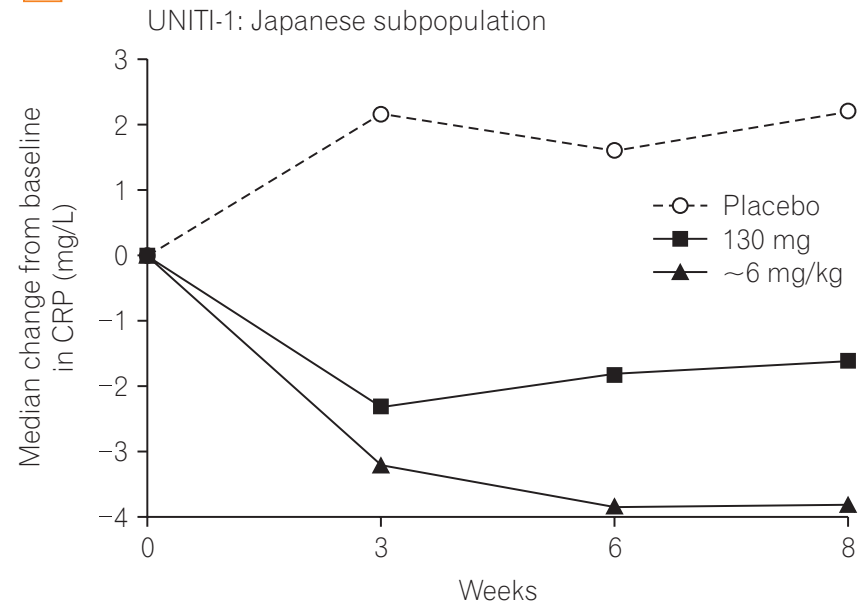

C

UNITI-2: Japanese subpopulation

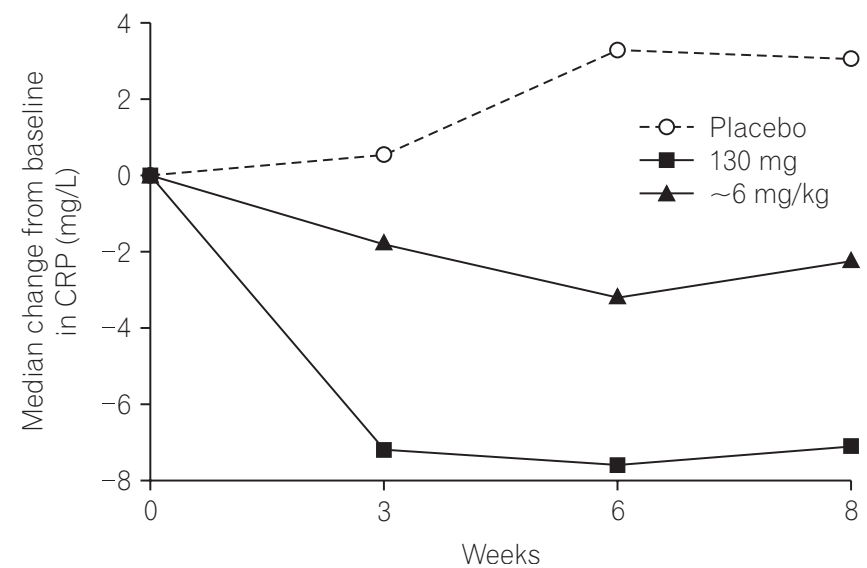

B

UNITI-1: overall population

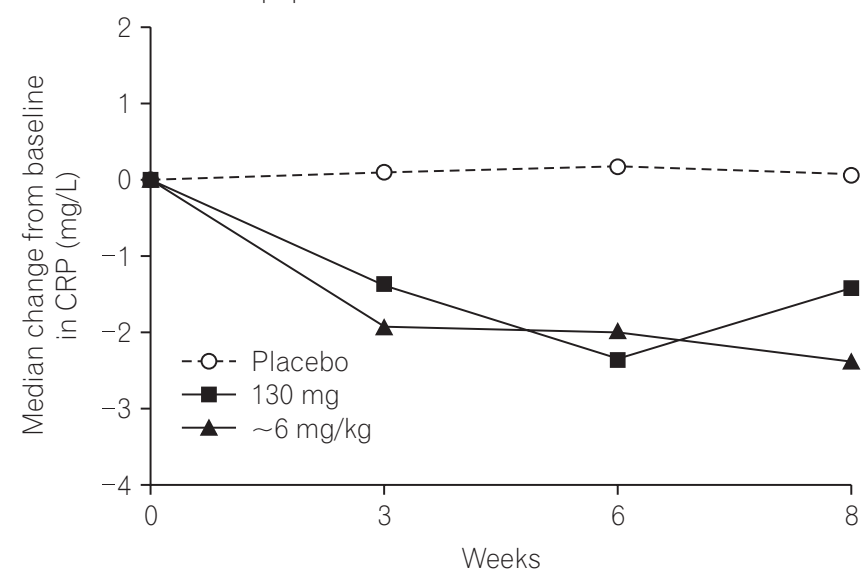

D

UNITI-2: overall population

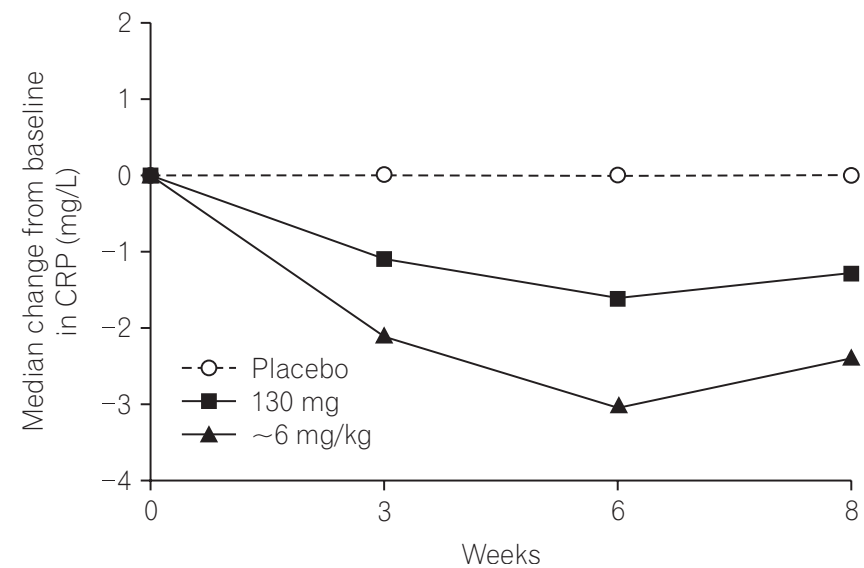

Supplementary Fig. 1. Change in CRP concentration through week 8 of induction studies. Panel B and D are adapted from Feagan BG, et al. N Engl J Med 2016;375:1946-1960, with permission Massachusetts Medical Society. ${ }^{19}$ 
See "Efficacy and safety of ustekinumab in Japanese patients with moderately to severely active Crohn's disease: a subpopulation analysis of phase 3 induction and maintenance studies" on page 475-486.
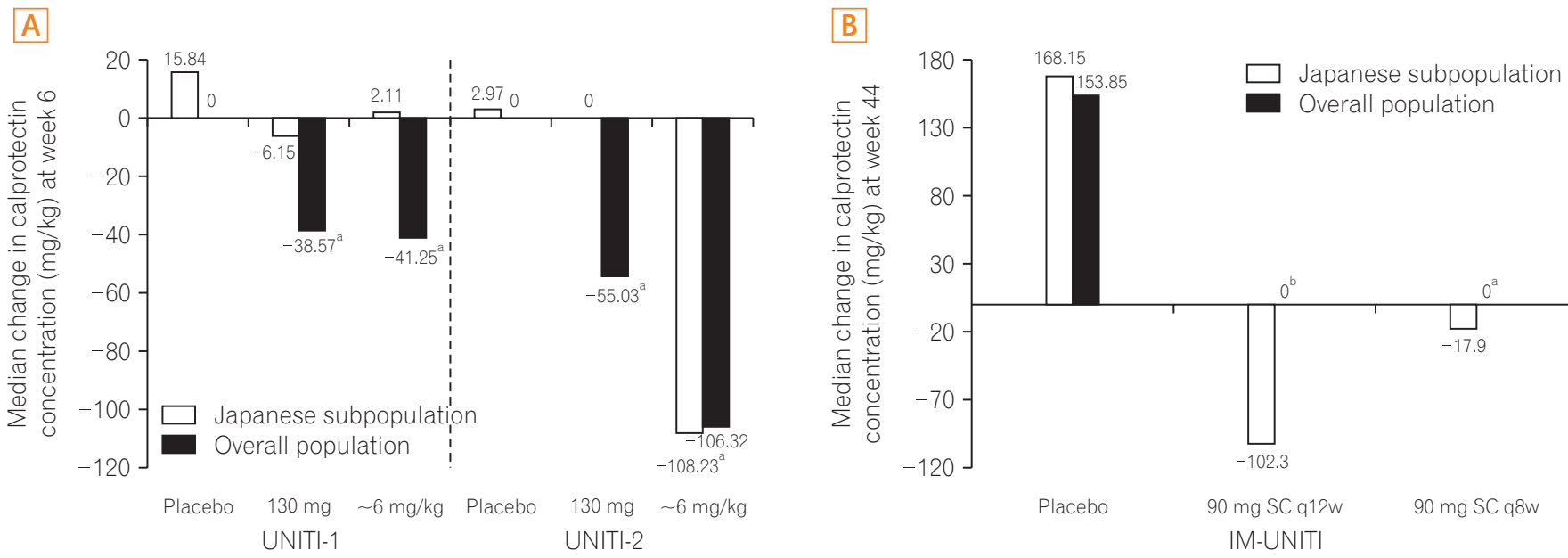

Supplementary Fig. 2. Change in fecal calprotectin concentration at: (A) week 6 of the induction studies; (B) week 44 of the maintenance study. ${ }^{\mathrm{a}} \mathrm{P}<0.001$ vs. placebo; ${ }^{b} P=0.002$ vs. placebo. $q 12 \mathrm{w}$, every 12 weeks; $\mathrm{q} 8 \mathrm{w}$, every 8 weeks. 
See "Efficacy and safety of ustekinumab in Japanese patients with moderately to severely active Crohn's disease: a subpopulation analysis of phase 3 induction and maintenance studies" on page 475-486.

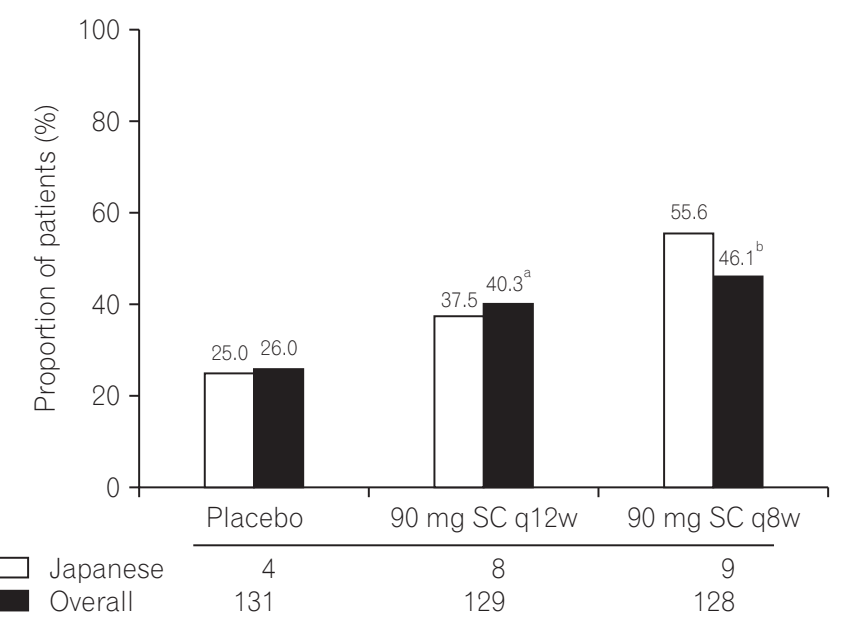

Supplementary Fig. 3. Sustained clinical remission at weeks 36, 40, and 44 of the maintenance study. ${ }^{a} P=0.023$ vs. placebo; ${ }^{b} P<0.001$ vs. placebo. q12w, every 12 weeks; q8w, every 8 weeks. 
See "Efficacy and safety of ustekinumab in Japanese patients with moderately to severely active Crohn's disease: a subpopulation analysis of phase 3 induction and maintenance studies" on page 475-486.

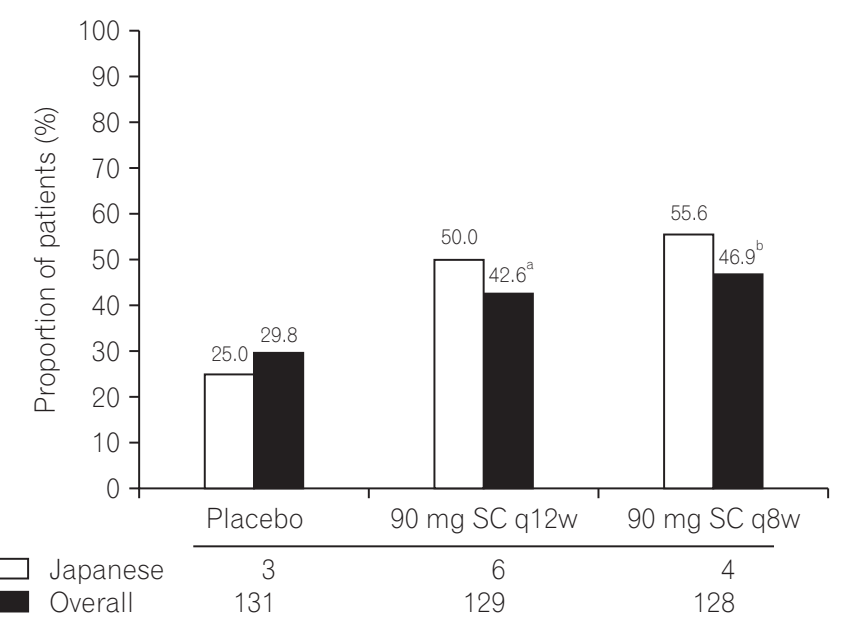

Supplementary Fig. 4. Corticosteroid-free remission at week 44 of the maintenance study. ${ }^{a} P=0.035$ vs. placebo but only nominally significant per the hierarchical testing procedure; ${ }^{b} P=0.004$ vs. placebo. $q 12 w$, every 12 weeks; $q 8 \mathrm{w}$, every 8 weeks. 
See "Efficacy and safety of ustekinumab in Japanese patients with moderately to severely active Crohn's disease: a subpopulation analysis of phase 3 induction and maintenance studies" on page 475-486.

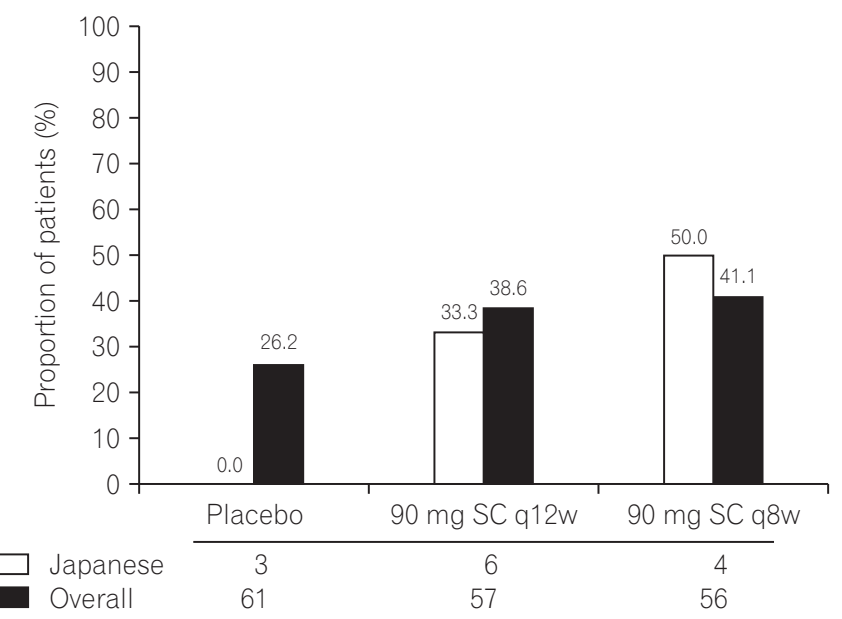

Supplementary Fig. 5. Clinical remission at week 44 in patients who were refractory or intolerant to tumor necrosis factor-antagonist therapy (UNITI-1 patients). q12W, every 12 weeks; q8w, every 8 weeks. 
See "Efficacy and safety of ustekinumab in Japanese patients with moderately to severely active Crohn's disease: a subpopulation analysis of phase 3 induction and maintenance studies" on page 475-486.

A

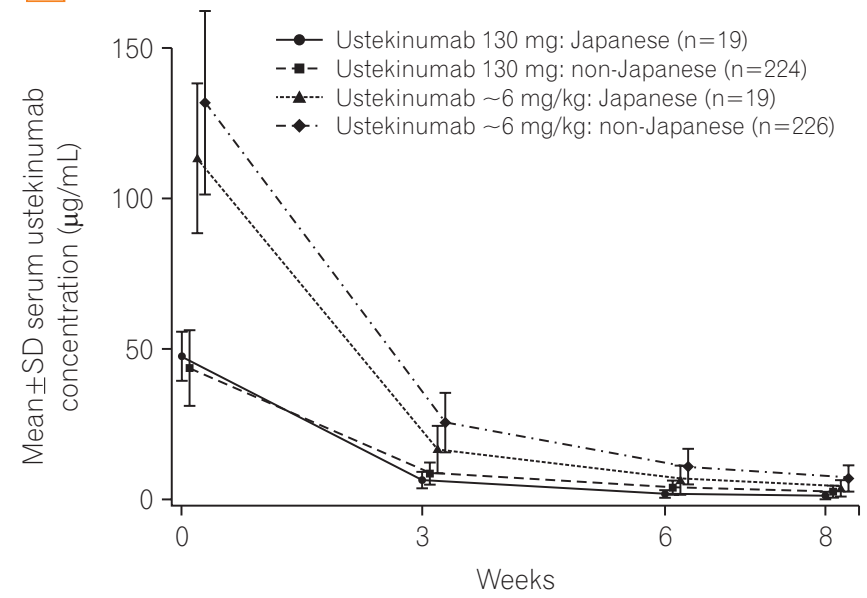

B

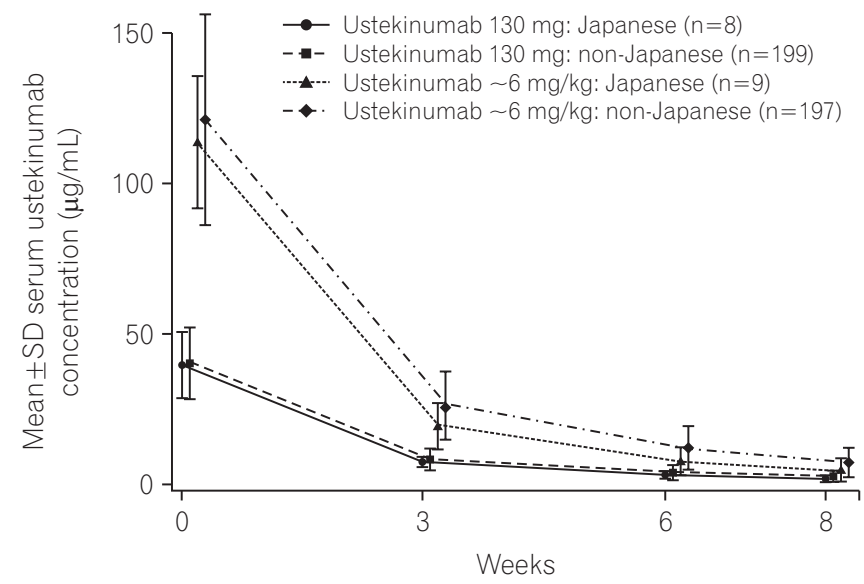

Supplementary Fig. 6. Mean \pm SD serum ustekinumab concentrations $(\mu \mathrm{g} / \mathrm{mL})$ through week $8(A)$ in UNITI-1 and (B) in UNITI-2 studies. 
See "Efficacy and safety of ustekinumab in Japanese patients with moderately to severely active Crohn's disease: a subpopulation analysis of phase 3 induction and maintenance studies" on page 475-486.
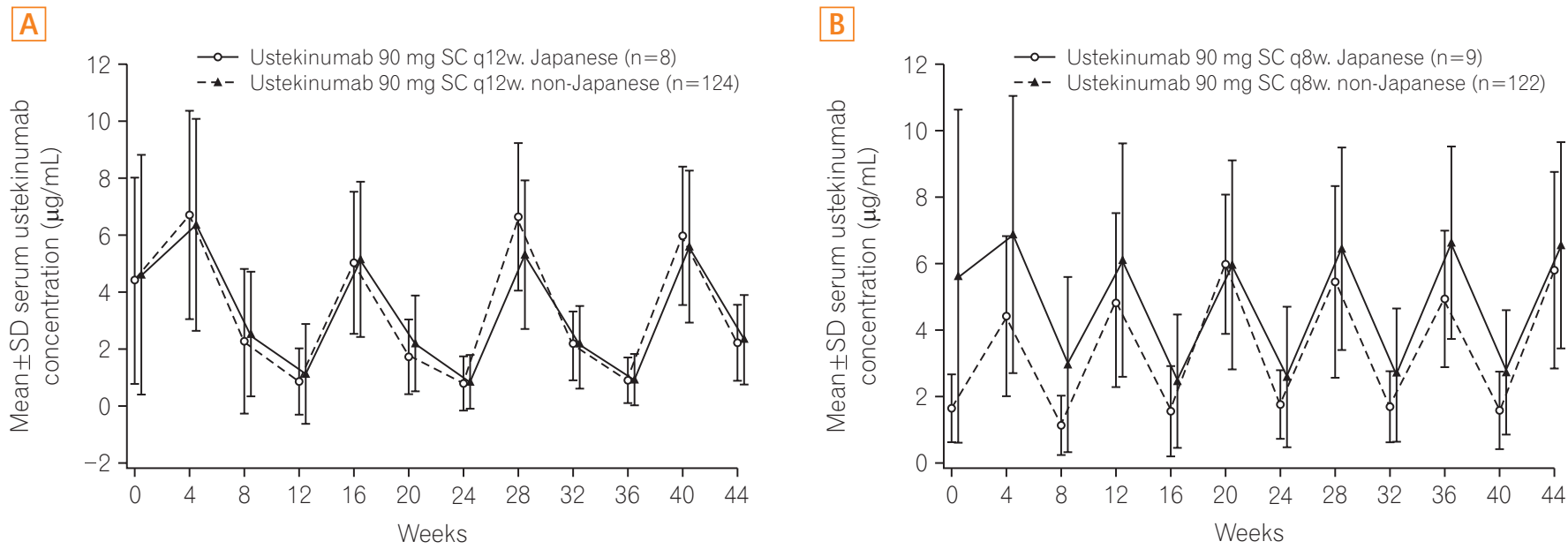

Supplementary Fig. 7. Mean \pm SD serum ustekinumab concentrations $(\mu \mathrm{g} / \mathrm{mL})$ in IM-UNITI (A) in the $90 \mathrm{mg} S \mathrm{SC} 12 \mathrm{w}$ group and (B) in the $90 \mathrm{mg}$ SC q8w group. q12w, every 12 weeks; q8w, every 8 weeks. 


\section{Supplementary Information}

\section{List of UNITI-1 investigators in Japan}

A Ando, Shiga University of Medical Science Hospital, Otsu, Shiga; T Ashida, Sapporo Higashi Tokushukai Hospital, Sapporo, Hokkaido; H Hanai, Hamamatsu South Hospital, Minamiku, Hamamatsu; T Ishida, Oita Red Cross Hospital, Oita City, Oita; H Ito, Kinshu-kai Infusion Clinic, Osaka City, Osaka; T Matsumoto, Kyushu University Hospital, Fukuoka city, Fukuoka; S Motoya, Hokkaido P.W.F.A.C. Sapporo-Kosei General Hospital, Sapporo-shi, Hokkaido; S Nakamura, Hyogo College of Medicine, Nishinomiya, Hyogo; Y Sameshima, Sameshima Hospital, Kagoshima-city, Kagoshima; Y Suzuki, Toho University, Sakura Medical Center, Sakura-shi, Chiba; K Watanabe, Osaka City University Hospital, Osaka City, Osaka; H Yamagami, Osaka City University Hospital, Osaka City, Osaka; T Yamamoto, Yokkaichi Social Insurance Hospital, Department of Surgery, Yokkaichi, Mie; K Yao, Fukuoka University Chikushi Hospital, Chikushi, Fukuoka.

\section{List of UNITI-2 Investigators in Japan}

T Ashida, Sapporo Higashi Tokushukai Hospital, Sapporo, Hokkaido; H Hanai, Hamamatsu South Hospital, Minamiku, Hamamatsu; N Horiki, Mie University Hospital, Mie Prefecture, Tsu; H Iijima, Osaka University Hospital Department of Medicine, Gastroenterology and Hepatology, Suita; T Ishida, Oita Red Cross Hospital, Oita City, Oita; H Ito, Kinshu-kai Infusion Clinic, Osaka City, Osaka; M Iwabuchi, National Hospital Organization Sendai Medical Center, Miyagi Prefecture, Sendai; T Kanai, Keio University Hospital, Shinjuku-ku, Tokyo; R Kunisaki, Yokohama City University Medical Center, Kanagawa, Minami-ku, Yokohama; A Maemoto, Sapporo Higashi Tokushukai Hospital, Sapporo, Hokkaido; K Matsuoka, Keio University Hospital, Shinjuku-ku, Tokyo; S Motoya, Hokkaido P.W.F.A.C. Sapporo-Kosei General Hospital, Sapporo-Shi, Hokkaido; S Nakamura, Hyogo College of Medicine 1-1 Mukogawacho, Nishinomiya, Hyogo; T Osada, Juntendo University Hospital, Tokyo; Y Sameshima, Sameshima Hospital, Kagoshima-city, Kagoshima; K Sugimoto, Hamamatsu University Hospital, Shizuoka Higashi-ku, Hamamatsu; S Tanaka, Hiroshima University Hospital, Hiroshima, Minami-ku; K Watanabe, Osaka City University Hospital, Osaka City, Osaka; H Yamagami, Osaka City University Hospital, Osaka City, Osaka; K Yao, Fukuoka University Chikushi Hospital, Chikushi, Fukuoka.

\section{List of IM-UNITI Investigators in Japan}

A Ando, Shiga University of Medical Science Hospital, Otsu, Shiga; T Ashida, Sapporo Higashi Tokushukai Hospital, Sapporo, Hokkaido; H Hanai, Hamamatsu South Hospital, Minamiku, Hamamatsu; H Iijima, Osaka University Hospital Department of Medicine, Gastroenterology and Hepatology, Suita; T Ishida, Oita Red Cross Hospital, Oita City, Oita; H Ito, Kinshu-kai Infusion clinic, Osaka City, Osaka; M Iwabuchi, National Hospital Organization Sendai Medical Center, Miyagi Prefecture, Sendai; R Kunisaki, Yokohama City University Medical Center, Kanagawa, Minami-ku, Yokohama; A Maemoto, Sapporo Higashi Tokushukai Hospital, Sapporo, Hokkaido; K Matsuoka, Kei University Hospital, Shinjuku, Tokyo; E Motohiro, Kyushu University Hospital, Fukuoka City, Fukuoka; S Motoya, Hokkaido P.W.F.A.C. Sapporo-Kosei General Hospital, Sapporo-shi, Hokkaido; S Nakamura, Hyogo College of Medicine, Nishinomiya, Hyogo; T Osada, Juntendo University Hospital, Tokyo; Y Sameshima, Sameshima Hospital, Kagoshima-city, Kagoshima; K Sugimoto, Hamamatsu University Hospital, Shizuoka Higashi-ku, Hamamatsu; Y Suzuki, Toho University, Sakura Medical Center, Sakura-shi, Chiba; K Takanori, Keio University Hospital, Shinjuku-ku, Tokyo; S Tanaka, Hiroshima University Hospital, Hiroshima, Minami-ku; K Watanabe, Osaka City University Hospital, Osaka City, Osaka; H Yamagami, Osaka City University Hospital, Osaka City, Osaka; T Yamamoto, Yokkaichi Hazu Medical Center, Yokkaichi, Mie; K Yao, Fukuoka University Chikushi Hospital, Chikushi, Fukuoka; K Yao, Fukuoka University Chikushi Hospital, Chikushi, Fukuoka.

\section{Dose adjustment in IM-UNITI}

In patients who subsequently met loss of response criteria at any time between week 8 and week 32 of maintenance, a singledose adjustment to ustekinumab $90 \mathrm{mg}$ q8w was done and patients were evaluated for 16 weeks for clinical improvement. If there was no improvement, study treatment was discontinued in these patients. Patients in IM-UNITI were allowed to continue treatment until week 272 (5 years) to further evaluate its long-term safety. 